\title{
Relación entre eventos cardiovasculares y un mundial de fútbol
}

\section{Erika Andrea Rincón-Escobar ${ }^{a}$, Sara Sofía Escobar-González ${ }^{a}$, Juan Esteban Gómez-Mesa ${ }^{\mathrm{b}, \mathrm{c}}$ y Juan Pablo Martínez-Cano ${ }^{\mathrm{d}, \mathrm{c}, *}$}

\author{
a Centro de Investigaciones Clínicas, Fundación Valle del Lili, Cali, Colombia \\ b Cardiología, Fundación Valle del Lili, Cali, Colombia \\ c Universidad Icesi, Cali, Colombia \\ d Ortopedia y Traumatología Deportiva, Fundación Valle del Lili, Cali, Colombia
}

Recibido el 15 de marzo de 2017; aceptado el 21 de julio de 2017

Disponible en Internet el 12 de noviembre de 2017

\author{
PALABRAS CLAVE \\ Fútbol; \\ Estrés; \\ Deportes; \\ Enfermedades \\ cardiacas
}

\begin{abstract}
Resumen
Introducción: el estrés causado por el fútbol puede tener efecto en la población general. Existen estudios con resultados controvertidos, tanto a favor de una asociación con mayor incidencia de eventos cardiovasculares, como en contra de ésta.

Objetivo: evaluar eventos cardiovasculares ocurridos durante el Mundial de fútbol Brasil 2014 en un centro de alto nivel de complejidad.

Métodos: estudio observacional, descriptivo, tipo corte transversal, en el que se analizan los registros de eventos cardiovasculares en el servicio de urgencias durante el Mundial de fútbol Brasil 2014 comparado con períodos control.

Resultados: 812 pacientes consultaron por eventos cardiovasculares en las fechas del Mundial y el período de control. En los días en los que jugó Colombia, hubo una incidencia de 0,91 veces la del período control (IC 95\%: 0,58-1,41; $\mathrm{p}=0,33$ ). Para los hombres, la incidencia de eventos cardiovasculares durante los días que jugó Colombia fue 0,88 veces la del período de control (IC 95\%: $0,47-1,62 ; \mathrm{p}=0,33$ ) y para las mujeres de 0,95 veces la del periodo control (IC 95\%: $0,48-1,84 ; p=0,44)$. Durante los días que jugó Colombia comparado con el período control, la tasa de incidencia para dolor torácico fue 0,94, para alteraciones del ritmo fue 0,90 y para el grupo de paro cardiaco 0,42 , sin significancia estadística.

Conclusión: no hubo mayor incidencia de eventos cardiovasculares en los días del mundial ni en los días en los que jugó el equipo colombiano; por el contrario, hubo disminución numérica en la incidencia de estos eventos.

(c) 2017 Sociedad Colombiana de Cardiología y Cirugía Cardiovascular. Publicado por Elsevier España, S.L.U. Este es un artículo Open Access bajo la licencia CC BY-NC-ND (http:// creativecommons.org/licenses/by-nc-nd/4.0/).
\end{abstract}

\footnotetext{
* Autor para correspondencia.

Correo electrónico: jpmartinezc@gmail.com (J.P. Martínez-Cano).
} 


\section{KEYWORDS}

Soccer;

Stress;

Sports;

Cardiovascular

disease

\section{Relationship between cardiovascular events and a World Cup}

\begin{abstract}
Introduction: Stress caused by football matches can have an effect on the general population. Reports about the association between soccer matches and cardiovascular diseases have been controversial.

Objective: To evaluate cardiovascular events during the 2014 World Cup at a high level of complexity centre in Cali-Colombia.

Methods: An observational, descriptive, cross-sectional study in which cardiovascular event records were analysed in the Emergency Department during the 2014 World Cup, and compared with control periods.

Results: A total of 812 patients were included for cardiovascular events on the World Cup dates vs control period. On days of matches involving the Colombian team, the incidence of cardiac

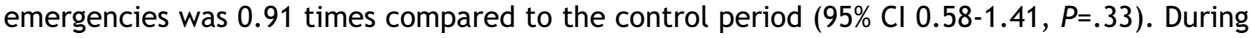
the days of matches played by the Colombian team, the incidence in male patients was 0.88 times compared to the control period $(95 \% \mathrm{Cl}: 0.47-1.62, P=.33)$ and the incidence in female patients was 0.95 times compared to the control period $(95 \% \mathrm{Cl}: 0.48-1.84, P=.44)$. During the 5 days in which Colombia played compared to the control period, the incidence rate for chest pain was 0.94 , for rhythm disturbances it was 0.90 , and 0.42 for cardiac arrest, with no statistically significant difference.

Conclusion: There was no greater incidence of cardiovascular events during the days of the World Cup or during the days of matches involving the Colombian team, although there was a tendency for a numerical decrease in incidence of these events.

(C) 2017 Sociedad Colombiana de Cardiología y Cirugía Cardiovascular. Published by Elsevier España, S.L.U. This is an open access article under the CC BY-NC-ND license (http:// creativecommons.org/licenses/by-nc-nd/4.0/).
\end{abstract}

\section{Introducción}

Además de los factores de riesgo clásicos para presentar un evento coronario, como sobrepeso, tabaquismo, hipertensión arterial o diabetes, desde hace varios años se ha descrito el papel que puede tener el estrés emocional en la aparición de este tipo de patologías ${ }^{1-5}$. Debido a la estimulación del sistema nervioso simpático y a la liberación de catecolaminas que ocurre durante el estrés, se produce aumento en la frecuencia cardiaca, presión arterial y demanda de oxígeno debido a mayor contractilidad ventricular. Esto a su vez contribuye a la ruptura de placas ateroscleróticas preexistentes ${ }^{6,7}$. Además, la estimulación de los receptores alfa en la circulación puede incrementar la resistencia vascular, la poscarga y la vasoconstricción coronaria, y así mismo aumentar la demanda de oxígeno y limitar el suministro de oxígeno al corazón. Por otro lado, el exceso de catecolaminas puede elevar la agregación plaquetaria y favorecer la aparición de arritmias cardiacas ${ }^{8}$.

En la literatura se han publicado estudios en los que se investigó la asociación entre competencias de fútbol y enfermedad cardiovascular o muerte por causas cardiovasculares en los espectadores o en la población general. Los resultados han sido variables, algunos a favor y otros en contra de esta asociación. En un estudio hecho en Alemania durante los días del mundial de fútbol de 2006, en el que participó el equipo alemán, la incidencia de emergencias cardiacas fue 2,66 veces la del grupo control ${ }^{9}$. Por el contrario, en un estudio realizado en población italiana durante tres competencias mundiales de fútbol, no encontraron un incremento en los infartos agudos de miocardio durante los días en los que hubo partidos que involucraran al equipo de ese país ${ }^{10}$.

El aumento en la incidencia de eventos cardiovasculares no ha sido reportado exclusivamente en los fanáticos del fútbol, también se ha observado en situaciones con un alto grado de estrés emocional como terremotos, desastres naturales $\mathrm{o}$ atentados terroristas ${ }^{11-13}$. En un estudio realizado en New Jersey después del atentado del 11 de septiembre, encontraron un incremento significativo en el número de pacientes con infarto agudo de miocardio ${ }^{12}$. En un estudio llevado a cabo en Japón después del terremoto de 2011 encontraron que todos los tipos de enfermedades cardiovasculares aumentaron independientemente de la edad, el sexo o el área de residencia ${ }^{13}$. Otro estudio hecho en bomberos, que analizó el riesgo de muerte según la actividad realizada, concluyó que actividades específicas como apagar incendios y responder a alarmas estuvieron asociados con un riesgo significativamente mayor comparado con actividades no relacionadas con emergencias ${ }^{14}$.

En Colombia, la población general podría tener un aumento en la incidencia de eventos cardiovasculares durante los días de partidos que involucraron la selección colombiana. Por esto, se investigó la asociación entre partidos de fútbol en las fechas en las que la selección Colombia participó en el Mundial Brasil 2014 y el ingreso hospitalario por eventos cardiacos en una institución de alto nivel de complejidad. 


\section{Métodos}

\section{Adquisición de datos}

Estudio observacional analítico tipo corte transversal de los registros del servicio de urgencias de una institución de alto nivel de complejidad. Los periodos de tiempo incluidos fueron del 12 al 13 de julio de 2014 y el periodo de control fue del 1 de junio al 31 de julio de 2012 y 2013, así como del 1 de junio al 11 de julio y del 14 al 31 de julio de 2014 .

Se incluyeron los pacientes ingresados a través del servicio de urgencias por algún evento cardiovascular según los códigos del CIE10. Se utilizaron los diagnósticos finales de la atención y los pacientes debían haber sido estudiados durante la misma. En los criterios de elegibilidad los pacientes debían ser mayores de 18 años, de cualquier género y que tuvieran una arritmia cardiaca, un infarto con o sin elevación del ST, angina estable o inestable, o paro cardiorrespiratorio. Se dividieron posteriormente en tres grupos: alteraciones del ritmo cardiaco, dolor torácico y paro cardiaco.

Se obtuvieron los registros de estadística del servicio de urgencias de la Institución base del estudio. A partir de estos registros, se evaluaron los siguientes datos: fecha de ingreso, edad, género y diagnóstico CIE10. Además, se evaluó si existían diferencias en el tipo de seguridad social y en las comorbilidades entre los grupos estudiados, como posibles factores de confusión.

El protocolo del estudio fue aprobado por el Comité de Ética en Investigación Biomédica de la Institución. Por el diseño retrospectivo del estudio con uso de información anónima, se omitió la necesidad de consentimiento informado.

\section{Análisis estadístico}

Se usó una regresión de Poisson para obtener el número de emergencias cardiovasculares por día. Se compararon los eventos ocurridos durante los tres diferentes periodos: los 5 días de partidos de la copa del mundo en los que jugó el equipo colombiano, los 27 días del mundial en los que no jugó Colombia y los 151 días de control.

Se calcularon las tasas de incidencia para los 5 días en los que hubo partidos de Colombia y los 27 días en los que no jugó dicho equipo, en comparación con los días del control. Luego se calcularon las tasas de incidencia por subgrupos de pacientes, de acuerdo con el diagnóstico, la edad y el género.

Los análisis se realizaron en el software STATA $13.0^{\circledR}$. Un valor de $\mathrm{P}$ menor de 0,05 fue considerado como significancia estadística.

\section{Resultados}

Se incluyeron en total 812 pacientes con eventos cardiovasculares. La figura 1 muestra el número de eventos por día. El mundial de fútbol de 2014 en Brasil inició el 12 de junio y finalizó el 13 de julio. Sólo uno de los 5 partidos en los que Colombia participó se asoció con incremento en el número de eventos cardiovasculares comparado con el periodo de control; sin embargo, en los otros 4 partidos en los que Colombia jugó, hubo menos número de eventos comparado con el grupo control.

La tabla 1 muestra las tasas de incidencia para eventos cardiovasculares. La incidencia durante los partidos que jugó Colombia fue 0,91 veces la del grupo control; pese a ello, no hubo una diferencia estadísticamente significativa. En el grupo de días del mundial en los que no jugó Colombia también hubo una incidencia menor respecto al grupo control $(0,76$ veces la del grupo control) sin significancia estadística.

La tabla 2 muestra las características de los pacientes que tuvieron un evento cardiovascular. Durante los 5 días que jugó Colombia, la proporción de pacientes hombres fue menor comparada con los días de control (52,3\% vs. 53,7\%). Para los hombres, la incidencia de eventos cardiovasculares durante los 5 días que jugó Colombia fue 0,88 veces la del periodo de control y para las mujeres de 0,95 veces la del periodo control, sin diferencias significativas en ninguno de los dos grupos $(p>0,05)$.

En los tres grupos incluidos, el promedio de edad fue similar, siendo un poco menor en los pacientes del grupo de los 5 días en los que jugó Colombia respecto al grupo control $(51,4$ vs. 53,1$)$.

Los diagnósticos CIE10 se dividieron en tres categorías (tabla 3). Durante los 5 días en los que jugó Colombia hubo 2,74 eventos de dolor torácico por día, comparado con 3,42 por día en el grupo control, lo que corresponde a una tasa de incidencia de 0,94 . Durante los 5 días en los que jugó, la tasa de incidencia de alteraciones del ritmo fue de 0,90 y para el grupo de paro cardiaco de 0,42. Ninguna de estas disminuciones en la incidencia fue estadísticamente significativa. En el periodo del mundial en los días que no jugó Colombia, la incidencia de paro cardiaco fue 0 comparada con el grupo control, con diferencia estadísticamente significativa $(p=0,003)$.

Como se aprecia en la tabla 4, no se encontraron diferencias significativas en el tipo de seguridad social ni en las comorbilidades entre los participantes de los tres grupos. La comorbilidad que más diferencia registró fue la enfermedad coronaria, más frecuente en el grupo control que en los otros grupos, sin embargo, sin diferencias significativas con los otros grupos de manera general $(p=0,49)$, ni tampoco al comparar la variable individualmente con cada grupo. No se encontró ningún factor de confusión asociado.

\section{Discusión}

En diferentes reportes de la literatura se ha demostrado asociación entre los partidos de fútbol y la mayor incidencia de eventos cardiovasculares. Un estudio adelantado en Alemania para el mundial de fútbol del 2006, mostró que los eventos cardiovasculares aumentaron significativamente en los días en los que el equipo alemán participó en algún partido (razón de incidencia [RI] 2,66; 95\% intervalo de confianza [IC], 2,33-3,04; $p<0,001$ ), sin embargo, en los otros días del mundial no reportaron aumento en los eventos cardiovasculares (RI: 1,$11 ; 95 \%$ IC: $0,99-1,25 ; p=0,08)^{9}$. En otro estudio realizado en 2014 para el mundial de fútbol de Brasil, reportaron un aumento significativo en los eventos cardiovasculares durante todos los días en los que se llevó a cabo la copa del mundo, con picos en los días en los que jugaba 


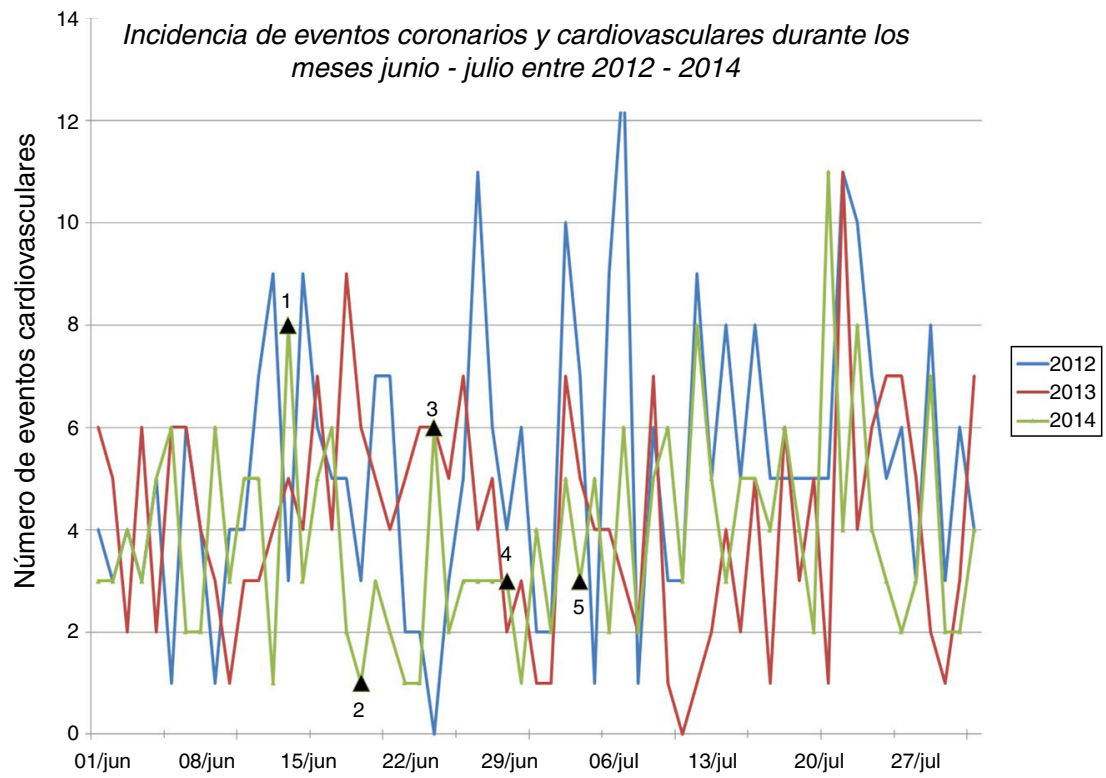

Figura 1 Incidencia de eventos coronarios y cardiovasculares durante los meses junio - julio entre 2012 - 2014.

Tabla 1 Tasas de incidencia para eventos cardiovasculares en los días del mundial de fútbol comparado con el periodo de control

\begin{tabular}{|c|c|c|c|c|}
\hline & $\begin{array}{l}\text { Total de } \\
\text { pacientes }\end{array}$ & $\begin{array}{l}\text { Eventos durante los } \\
5 \text { días que jugó Colombia } \\
(\mathrm{n}=21)\end{array}$ & $\begin{array}{l}\text { Eventos durante los } \\
27 \text { días del mundial que } \\
\text { no jugó Colombia }(n=94)\end{array}$ & $\begin{array}{l}\text { Eventos durante los } \\
151 \text { días de control } \\
(\mathrm{n}=696)\end{array}$ \\
\hline Eventos por día & 812 & 4,2 & 3,5 & 4,6 \\
\hline $\begin{array}{l}\text { Razón de } \\
\text { incidencia ( } 95 \% \text { IC) }\end{array}$ & & $0,91(0,58-1,41)$ & $0,76(0,47-1,20)$ & 1 \\
\hline Valor de $p$ & & 0,33 & 0,11 & \\
\hline
\end{tabular}

Tabla 2 Características de los pacientes que tuvieron eventos cardiovasculares durante la copa del mundo comparado con el tiempo de control

\begin{tabular}{|c|c|c|c|c|}
\hline Características & $\begin{array}{l}\text { Total de } \\
\text { pacientes }\end{array}$ & $\begin{array}{l}\text { Eventos durante los } \\
5 \text { días que jugó Colombia } \\
(n=21)\end{array}$ & $\begin{array}{l}\text { Eventos durante los } \\
27 \text { días del mundial que } \\
\text { no jugó Colombia }(n=94)\end{array}$ & $\begin{array}{l}\text { Eventos durante los } \\
151 \text { días de control } \\
(\mathrm{n}=696)\end{array}$ \\
\hline Sexo masculino & 435 & & & \\
\hline Porcentaje de pacientes & & 52,3 & 53,1 & 53,7 \\
\hline $\begin{array}{l}\text { Razón de incidencia } \\
(95 \% \text { IC) }\end{array}$ & & $0,88(0.47-1,62)$ & $0,76(0,39-1,43)$ & 1 \\
\hline Valor de $\mathrm{p}$ & & 0,33 & 0,18 & \\
\hline Sexo femenino & 377 & & & \\
\hline Porcentaje de pacientes & & 47,6 & 46,8 & 46,3 \\
\hline $\begin{array}{l}\text { Razón de incidencia } \\
(95 \% \text { IC) }\end{array}$ & & $0,95(0,48-1,84)$ & $0,76(0,37-1,53)$ & 1,0 \\
\hline Valor de $\mathrm{p}$ & & 0,4 & 0,2 & \\
\hline Edad-Años & 812 & $51,4 \pm 22,5$ & $52,4 \pm 20,2$ & $53,1 \pm 21,3$ \\
\hline
\end{tabular}

Las tasas de incidencia se calcularon con el promedio de eventos cardiovasculares por día del mundial sobre el promedio de eventos cardiovasculares por día durante el tiempo de control. 
Tabla 3 Tasas de incidencia de eventos cardiovasculares en los días del mundial comparado con los días de control de acuerdo con el grupo diagnóstico

\begin{tabular}{|c|c|c|c|c|}
\hline Diagnósticos & $\begin{array}{l}\text { Total de } \\
\text { pacientes }\end{array}$ & $\begin{array}{l}\text { Eventos durante los } \\
5 \text { días que jugó Colombia } \\
(\mathrm{n}=21)\end{array}$ & $\begin{array}{l}\text { Eventos durante los } \\
27 \text { días del mundial que } \\
\text { no jugó Colombia }(n=94)\end{array}$ & $\begin{array}{l}\text { Eventos durante los } \\
151 \text { días de control } \\
(\mathrm{n}=696)\end{array}$ \\
\hline Dolor torácico & 606 & & & \\
\hline Porcentaje de pacientes & & 76,1 & 78,7 & 74,1 \\
\hline $\begin{array}{l}\text { Razón de incidencia } \\
(95 \% \text { IC) }\end{array}$ & & $0,94(0,56-1,57)$ & $0,79(0,46-1,35)$ & 1,0 \\
\hline Valor de $\mathrm{p}$ & & 0,4 & 0,18 & \\
\hline Alteraciones del ritmo & 194 & & & \\
\hline Porcentaje de pacientes & & 23,8 & 20,2 & 24,3 \\
\hline $\begin{array}{l}\text { Razón de incidencia } \\
(95 \% \text { IC) }\end{array}$ & & $0,90(0,34-2,35)$ & $0,63(0,20-1,79)$ & 1 \\
\hline Valor de $p$ & & 0,41 & 0,17 & \\
\hline Paro cardiaco & 12 & & & \\
\hline Porcentaje de pacientes & & 0,03 & 0,00 & 0,07 \\
\hline $\begin{array}{l}\text { Razón de incidencia ( } 95 \% \\
\text { IC) }\end{array}$ & & $0,42(0,071-1,87)$ & $0(0-0,69)$ & 1 \\
\hline Valor de $\mathrm{p}$ & & 0,11 & 0,003 & \\
\hline
\end{tabular}

Tabla 4 Relación entre los tres grupos con la seguridad social y los antecedentes médicos

\begin{tabular}{|c|c|c|c|c|}
\hline & Mundial cuando Colombia jugó & Mundial de fútbol & Control & Valor de $p$ \\
\hline \multicolumn{5}{|l|}{ Régimen de salud } \\
\hline Privado (\%) & 33,33 & 41,66 & 50 & 0,53 \\
\hline EPS (\%) & 52,38 & 52,77 & 45,23 & \\
\hline Subsidiado (\%) & 14,28 & 5,55 & 4,76 & \\
\hline \multicolumn{5}{|l|}{ Antecedentes } \\
\hline HTA (\%) & 42,85 & 38,88 & 35,71 & 0,86 \\
\hline $\mathrm{DM}(\%)$ & 9,52 & 2,77 & 11,9 & 0,32 \\
\hline Enfermedad coronaria (\%) & 9,52 & 8,33 & 16,66 & 0,49 \\
\hline Arritmias (\%) & 9,52 & 19,44 & 16,66 & 0,61 \\
\hline Dislipidemia (\%) & 14,28 & 13,88 & 11,9 & 0,95 \\
\hline
\end{tabular}

Las tasas de incidencia se calcularon con el promedio de eventos cardiovasculares por día del mundial, sobre el promedio de eventos cardiovasculares por día durante el tiempo de control.

EPS: entidad promotora de salud, HTA: hipertensión arterial, DM: diabetes mellitus.

el equipo brasilero ${ }^{15}$. Nuestro estudio no mostró una asociación entre el aumento de eventos cardiovasculares durante los días del mundial de fútbol Brasil 2014, ni en los días en los que hubo partido de Colombia; contrario a esto, se mostró una tendencia a menor incidencia de estos eventos.

Respecto al género, en diferentes estudios se ha reportado que los hombres son más susceptibles a tener un evento cardiovascular por el estrés emocional secundario a un partido de fútbol. En el estudio de Wilbert-Lampen et al. la mayor proporción de eventos cardiovasculares durante los días que jugó el equipo alemán fueron hombres ${ }^{9}$ similar a lo reportado por Carroll et al. en 2002 cuando examinaron las admisiones hospitalarias en los días de la Copa mundial de fútbol de $1998^{16}$ y por Witte et al. en el año 2000, quienes reportaron que la mortalidad por enfermedad coronaria aumentó en hombres en el día del partido en el que Alemania fue eliminado de la Copa de fútbol europea ${ }^{17}$. Los hallazgos de nuestro estudio, en contra de alguna asociación del fútbol con eventos cardiovasculares, se mantuvieron aún durante el análisis de los datos por género.

En cuanto al análisis por grupos de diagnóstico, en el grupo específico de paro cardiaco se encontró menor incidencia de eventos en los días del mundial comparados con el grupo control (RI: 0, 95\% IC: 0-0,69. $p=0,003$ ), contrario a lo encontrado en el estudio de Wilbert-Lampen et al. quienes reportaron mayor número de eventos por día de infarto agudo de miocardio con elevación y sin elevación del ST y arritmias cardiacas con síntomas mayores y menores en los días en los que el equipo alemán participó en un partido, comparado con el grupo de periodo control, todos con diferencias estadísticamente significativas ${ }^{9}$.

Pese a que los hallazgos de nuestro estudio no muestran diferencias estadísticamente significativas, son comparables con publicaciones que han descartado alguna asociación 
entre el fútbol y un aumento de los infartos de miocardio u otros eventos cardiovasculares. Entre estos reportes, un estudio de Barone-Adesi et al., en el que se incluyó la población italiana durante el Mundial del 2002, la Eurocopa 2004 y el Mundial 2006 no reportó aumento de los eventos cardiovasculares cuando su selección nacional jugaba (riesgo relativo combinado 1,$01 ; 95 \%$ IC: $0,98-1,05)^{10}$. Otra publicación realizada en Alemania sobre el mundial de fútbol del 2006, concluyó que ver fútbol no estuvo asociado con un incremento en la incidencia de eventos cardiovasculares, sin importar si el equipo alemán jugaba o no ${ }^{18}$. Resultados similares han sido reportados en Portugal en un estudio publicado en 2011 en el que no encontraron diferencias en el número de infartos por día cuando jugaba el equipo de ese país ${ }^{19}$.

Aspectos que pueden tener influencia en los diferentes resultados de la literatura son los reportados por Leeka et al. quienes mostraron que la relación entre eventos deportivos y el aumento de la admisión hospitalaria por causa cardiaca depende de diferentes variables como la importancia del partido, la intensidad del juego y la filiación regional con el deporte $^{20}$. Es posible que para la población colombiana no haya expectativas tan importantes con la selección nacional, ya que no ha conseguido hasta ahora títulos en una copa mundo de mayores y que por eso no haya tanto estrés alrededor de sus partidos de fútbol. Podría ser que el comportamiento sea diferente cuando se trata del equipo local de fútbol en el torneo nacional, donde hay mayores probabilidades y expectativas de alcanzar el campeonato. Una situación de estrés más cercana a lo real podría aumentar el riesgo de este tipo de eventos, pero eso es materia de futuros estudios.

La principal limitación de nuestro estudio es que aunque los desenlaces se evalúan en el periodo de tiempo del mundial y en las fechas en que jugó la selección colombiana de fútbol, no se conoce con exactitud si los participantes eran espectadores o no del fútbol. Por lo tanto, no se puede hacer una asociación de causalidad entre el mundial y los desenlaces de interés. Sin embargo, a nivel poblacional, sí es posible evaluar la asociación entre ambos fenómenos, tal y como se ha hecho en este estudio. Se requerirán estudios adicionales más adelante para establecer si hay o no una relación de causalidad.

Hay otras posibles limitaciones como el sesgo de selección que podría haber en relación a que se trate de un único centro médico. Aunque se trata de un centro que atiende población de la mayoría de los seguros, puede haber mayor tendencia hacia los seguros del régimen contributivo y privado. Por otro lado, siendo un estudio retrospectivo, que utilizó los diagnósticos del CIE 10, para algunos pacientes podría haber dificultad en la definición exacta del diagnóstico debido a que la codificación puede ser un reto en ocasiones. Sin embargo, no hay razones para pensar que esta dificultad sería específica para ciertos días del año en particular, por lo que no consideramos que pueda afectar el objetivo principal del estudio de comparar las consultas por ciertos diagnósticos en los días del mundial y en los días en los que no lo hubo.

Los resultados de este estudio sugieren que en Colombia no hubo riesgo aumentado de eventos cardiovasculares durante el mundial de fútbol Brasil 2014. Incluso, contrario a esto, este tipo de desenlaces podría ser más bajo durante un evento deportivo como el mundial de fútbol.

\section{Responsabilidades éticas}

Protección de personas y animales. Los autores declaran que para esta investigación no se han realizado experimentos en seres humanos ni en animales.

Confidencialidad de los datos. Los autores declaran que en este artículo no aparecen datos de pacientes.

Derecho a la privacidad y consentimiento informado. Los autores declaran que en este artículo no aparecen datos de pacientes.

\section{Financiación}

Ninguna.

\section{Conflicto de intereses}

Ninguno.

\section{Bibliografía}

1. Leor J, Poole WK, Kloner RA. Sudden Cardiac Death Triggered by an Earthquake. N Engl J Med. 1996;334:413-9. Massachusetts Medical Society.

2. Meisel SR, Kutz I, Dayan KI, Pauzner H, Chetboun I, Arbel Y, et al. Effect of Iraqi missile war on incidence of acute myocardial infarction and sudden death in Israeli civilians. Lancet. 1991;338:660-1.

3. Serra Grima R, Carreño MJ, Tomás Abadal L, Brossa V, Ligero C, Pons J. Acute coronary events among spectators in a soccer stadium. Rev Esp Cardiol (English Ed). 2005;58:587-91.

4. Steptoe A, Kivimaki M. Stress and cardiovascular disease: an update on current knowledge. Annu Rev Public Heal. 2013;34:337-54.

5. Niiyama M, Tanaka F, Nakajima S, Itoh T, Matsumoto T, Kawakami $M$, et al. Population-based incidence of sudden cardiac and unexpected death before and after the 2011 earthquake and tsunami in Iwate, northeast Japan. J Am Hear Assoc. 2014;3(e000798):1-8.

6. Tofler GH, Stone PH, Maclure M, Edelman E, Davis VG, Robertson $\mathrm{T}$, et al. Analysis of possible triggers of acute myocardial infarction (the MILIS study). Am J Cardiol. 1990;66:22-7.

7. Chi JS, Kloner R. Stress and myocardial infarction. Heart. 2003;89:475-6.

8. Kloner RA, McDonald SA, Leeka J, Poole WK. Role of age, sex, and race on cardiac and total mortality associated with super bowl wins and losses. Clin Cardiol. 2011;34:102-7.

9. Wilbert-Lampen U, Leistner D, Greven S, Pohl T, Sper S, Völker C, et al. Cardiovascular events during World Cup soccer. N Engl J Med. 2008;358:475-83.

10. Barone-Adesi F, Vizzini L, Merletti F, Richiardi L. It is just a game: Lack of association between watching football matches and the risk of acute cardiovascular events. Int J Epidemiol. 2010;39:1006-13.

11. Stalnikowicz R, Tsafrir A. Acute psychosocial stress and cardiovascular events. Am J Emerg Med. 2002;20:488-91.

12. Allegra JR, Mostashari F, Rothman J, Milano P, Cochrane DG. Cardiac Events in New Jersey After the September 11, 2001, Terrorist Attack. New York. 2005;82:358-63. 
13. Aoki T, Fukumoto Y, Yasuda S, Sakata Y, Ito K, Takahashi J, et al. The Great East Japan Earthquake Disaster and cardiovascular diseases. Eur Heart J. 2012;33:2796-803.

14. Kales S, Soteridades E, Christophi C, Christiani D. Emergency Duties and deaths from heart disease among firefighters in the United States. N Engl J Med. 2007;356:1207-15.

15. Borges DGS, Monteiro RA, Schmidt A, Pazin-Filho A. World soccer cup as a trigger of cardiovascular events. Arq Bras Cardiol. 2013; 100:546-52.

16. Carroll D, Ebrahim S, Tilling K, Macleod J, Smith GD. Evidence that really matters Admissions for myocardial infarction and World Cup football: database survey. BMJ. 2002;325:1439-42.
17. Witte DR, Bots ML, Hoes AW, Grobbee DE. Cardiovascular mortality in Dutch men during 1996 European football championship: longitudinal population study. BMJ. 2000;321:1552-4.

18. Niederseer D, Thaler CW, Egger A, Niederseer MC, Plöderl M, Niebauer J. Watching soccer is not associated with an increase in cardiac events. Int J Cardiol. 2013;170:189-94.

19. Marques-Vidal P, Paccaud F. Watching footbal matches and the risk of acute myocardial infarction. Int J Epidemiol. 2010;39:13.

20. Olsen P, Elliott J, Frampton C, Bradley P. Winning or losing does matter: Acute cardiac admissions in New Zealand during Rugby World Cup tournaments. Eur J Prev Cardiol. 2015;22: 1254-60. 